الإدارة البيئية بشكل عام هي أسلوب منهجي لدمج الاعتبارات البيئية في المحميات الطبيعية مع الحركة السياحية فيها،

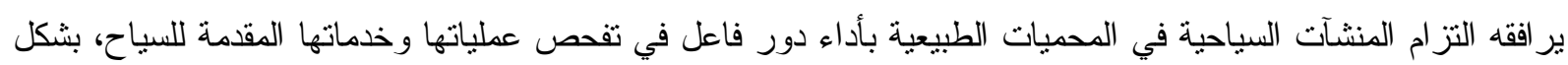

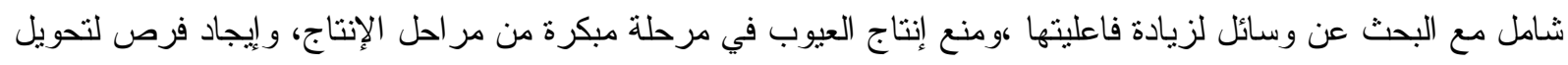

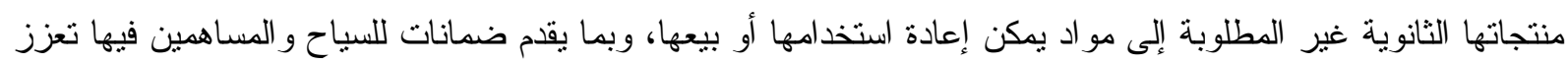
الثقة بها وبمنتجاتها . تحاول هذه الدر اسة النطرق إلى ماهية السلسلة القياسية الدولية ISO IS000 و الصادرة عن منظمة المو اصفات الدولية

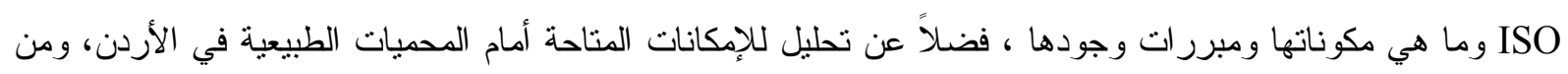

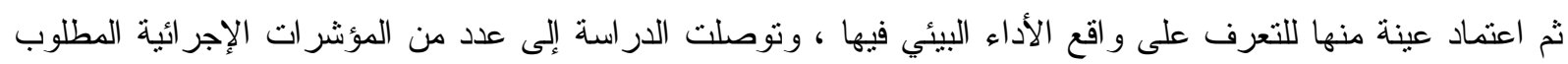
التصدي لها باتجاه تحسين مسنوى الأداء البيئي لتلك المحميات الطبيعية، ونم نلك من خلال خمسة محاور ترتبط بماهية السلسلة 14000 ISO ومبررات وجودها، ومكوناتها ، و الإمكانات المتاحة أمام المحميات الطبيعية في الأردن، كما تتاول

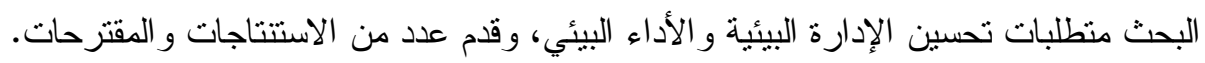
الكلمات المفتاحية: السلسلة القياسية الدولية، السياحة البيئية، صناعة السياحة، الأداء البيئي.

بالسلسلة القياسية الدولية 14000 ISO لنظم الإدارة البيئية في العام (Environmental Management Systems) كو 199 واكتمل الإصدار النهائي لها مع فاية عام 1997. تساؤلات الار اسة والأهداف.

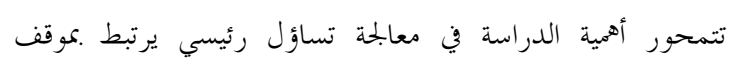

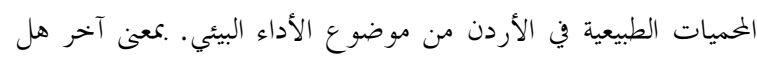

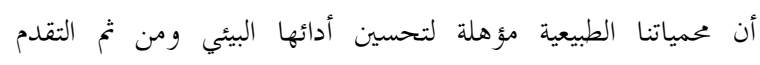

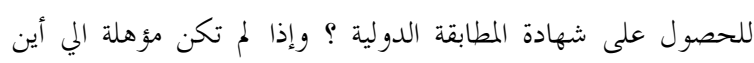
سيتم توجيه الجهود لتحقيق هدف التأهيل ؟ لـان

\section{المقدمة}

يعد مؤتمر البيئة والتنمية في ريو دي جانيرو عام بو 199 من المؤتمرات

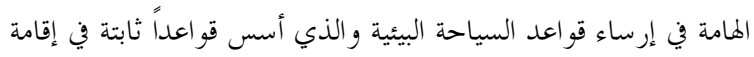

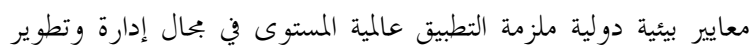
مواقع السياحة البيئية في العالم. وتشير القراءات في هذا الصدد إلى أن منظمة المواصفات الدولية

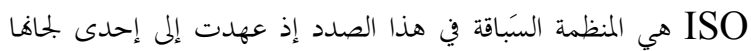
وهي اللجنة التقنية (TC 207) مسؤولية تصميم سلسلة من المعايير

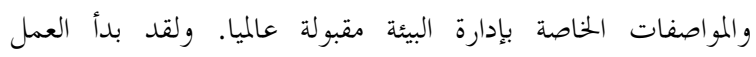




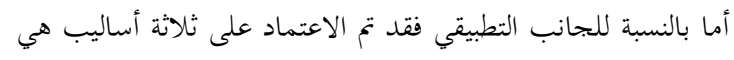

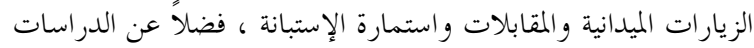

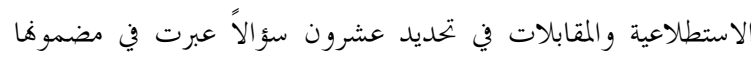

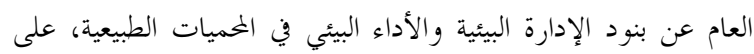

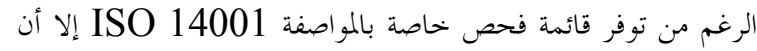

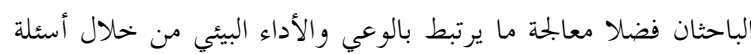

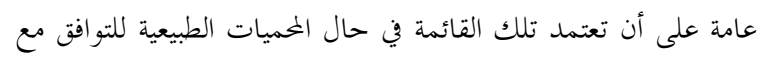

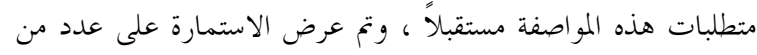

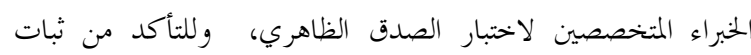

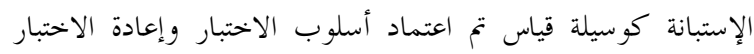

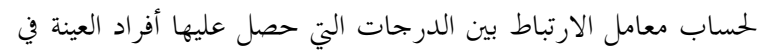

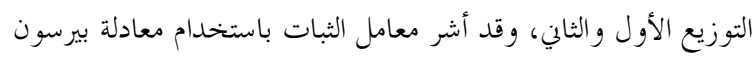

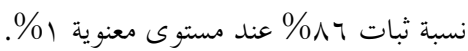

أولاً : ماهية السلسة ISO ISO 14000 ومبررات استخذامها في إدارة المحميات الطبيعية

هي سلسلة مواصفات قياسية دولية خاصة بنظم الإدارة

Environmental Management Systems البيئ تتكون من عدد من المواصفات تتمحور حول كل واحدة (EMS) منها بجموعة من البنود والمتطلبات ذات الطابع الفني والإداري ، الغاية

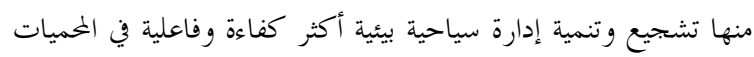

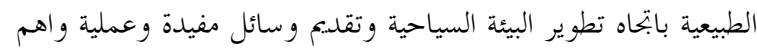

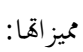
ا ب. اعلية الإدارة البيئية في المواقع السياحية.

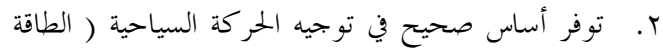

$$
\text { الاستيعابية ). }
$$

r. ت توفير تنمية سياحية مستدامة في الخميات الطبيعية.

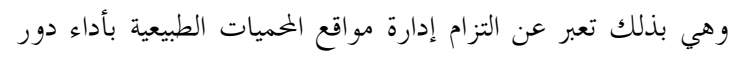

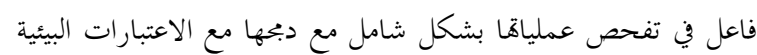

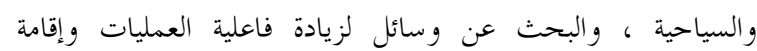
الدراسات الكفيلة للحد من إلحاق الضرر بمواقع السياحة البيئية في

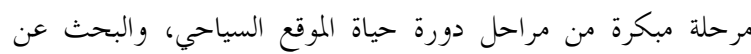

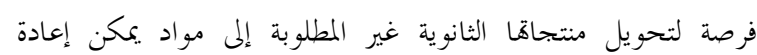
استخدامها و.ما يقدم للسياح والجهات ذات العلاقة بالقضايا البيئية ضمانات تعزز الثقة ها وبالخدمات السياحية المقدمة فيها.
هدف الدراسة إلى تعزيز أبعاد مفاهيم الإدارة البيئية في المواقع

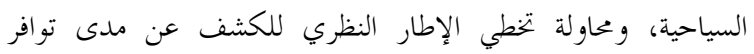

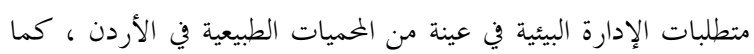
يهدف البحث إلى مناقشة واقع هذه الخميات الطبيعية فيما يرتبط

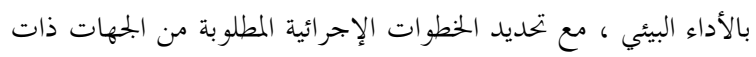

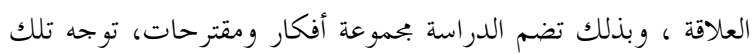
الخميات الطبيعية إلى الخطوة رقم واحد في مراحل تحسين الأداء البيئي

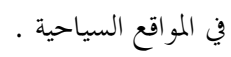

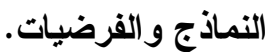

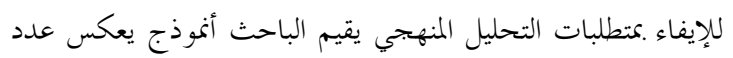

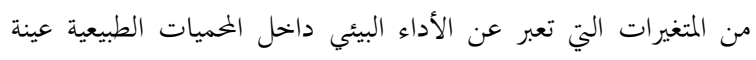

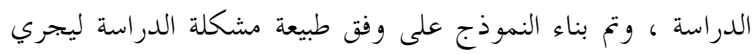
بعدها إقامة فرضية رئيسية مفادها : " تو اجه الحميات الطبيعية الأردنية ضعفاً في تغطيتها لمتطلبات الإدارة

$$
\text { البيئية وأسس تحسين الأداء البيئي في المواقع السياحية ". }
$$

\section{القطاع و العينة المختارة}

لتحقيق هدف الدراسة تم اختيار عينة نظامية من عدد من الخميات الطبيعية في الأردن وهي محمية دبين ،الشومري ، الأزرق المائية، ،البتراء،

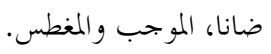
وتعد الخميات الطبيعية المختارة ذات مواقع سياحية مؤهلة من حيث الميثل

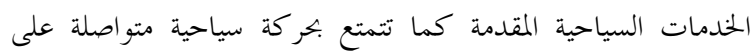

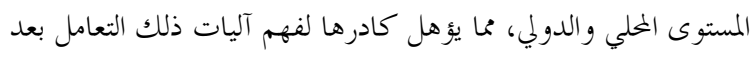

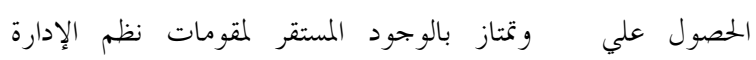
والمالية. 14001 شعادة أساليب جمع البياتات يري العديد من الباحثين بأن حث الإدارة العليا في الخميات الطبيعية

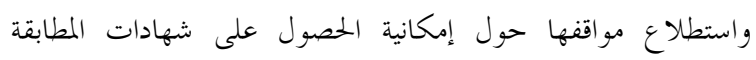

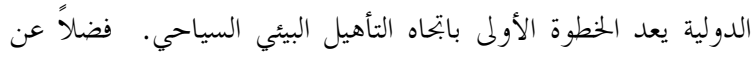

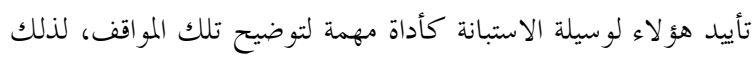

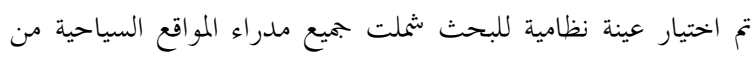
رئيس بجلس الإدارة ومدير الخمية ورؤساء الأقسام وإداريين وفنيين.

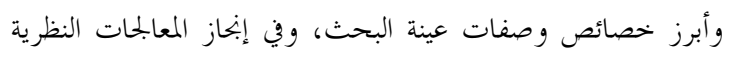

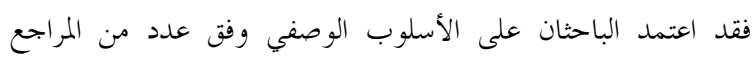

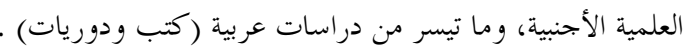




$$
\text { اقتصاديات السوق فعقلية الأخير ذات رؤية قصيرة النظر }
$$

\section{ISO ثانياً : مكونات السلسة 14000}

تضم سلسلة المواصفات القياسية الدولية 14000 ISO

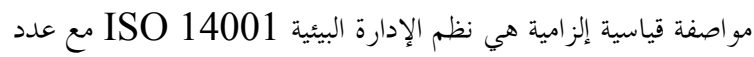

من المواصفات القياسية الإرشادية المكتملة التوثيق وكالآتي:

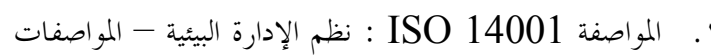

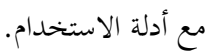
r. المواصفة ISO ISO نظم الإدارة البيئة - أدلة عامة عن مبادئ نظم التقنية الساندة لإدارة البيئة. r. المواصفة ISO IS010 أدلة للتدقيق والمراجعة البيئية - 140 مبادئ عامة للمراجعة الموضوعية، الاستقلالية، كفاءة المراجع، التطبيق المنهجي لإجراءات التقييم، اعتماد النتائج). ع. المواصفة ISO IS012 : أدلة التدقيق والمراجعة البيئية إجراءات التدقيق الجزء الأول : تدقيق نظم الإدارة البيئية

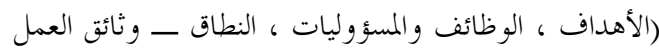
،جمع البيانات ، مراجعة النتائج ، إعداد التقارير). ه. المواصفة ISO ISO 14012 : أدلة التدقيق والمراجعة البيئية : Environmental معايير الكفاءة للمدققين في بحال البيئة Auditors

أو من خلال العمل ، كفاءة وصفات ومهارات المراجع).

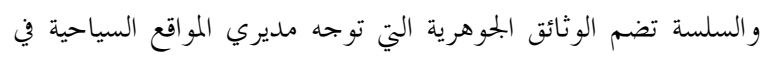

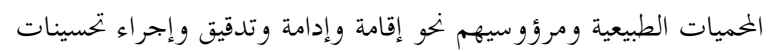

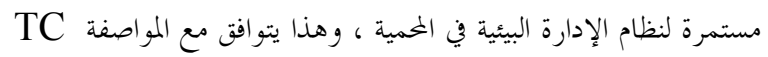
207

$$
\begin{aligned}
& \text { ، Labels المواصفة } 14020 \text { ISO العلامات } \\
& \text { التصريحات Declarations البيئة : وهي مبادئ أساسية } \\
& \text { لكل العلامات البيئية . } \\
& \text { r. المواصفة ISO ISO } 14021 \text { : العلامات (التصريحات) البيئية : } \\
& \text { البيان الذاتي Self declaration ، الشكاوى البيئية } \\
& \text { (المصطلحات و التعريفات) . }
\end{aligned}
$$

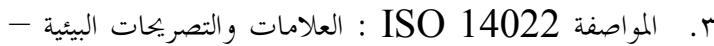

$$
\begin{aligned}
& \text { ع. ـ المواصفة } 14023 \text { ISO : العلامات والتصريحات البيئية - مكاجن } \\
& \text { طر ائق التحقق والاختبار. }
\end{aligned}
$$

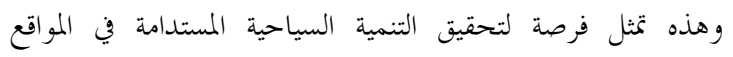

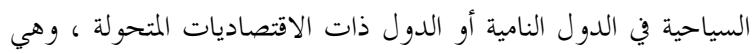

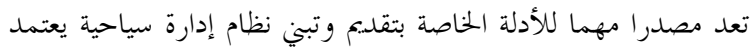

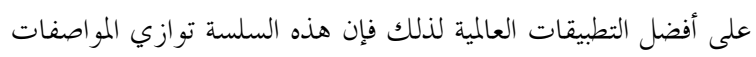
الدولية سلسة الموصفات الدولية 9000 ISO التي تمثل أداة لنقل

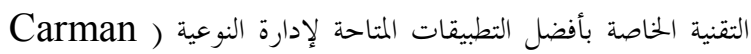
. 2007 والنيجة النهائية التي تسعى نوها هذه السلسة هي تطوير الأداء البيئي (Environmental Performance) في في المميات الطبيعية ـ وييرري التأكيد عالمياً على هذه السلسة كوفا تمثل مصدراً مرشداً نحو تبني نظام كفء وفاعل لإدارة البيئة في الحميات الطبيعية

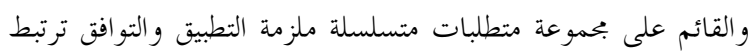

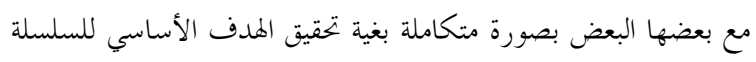

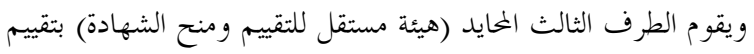

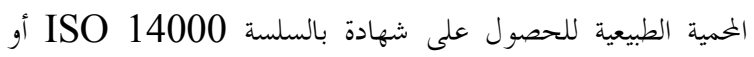
إحدى مواصفاةًا (Dixon 2005).

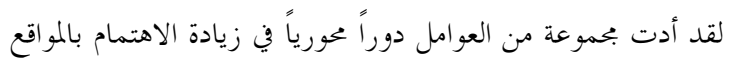

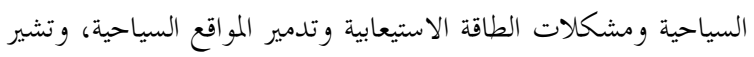

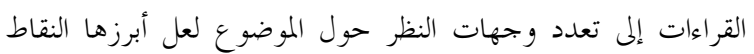

1. التحولات التاريخية في مواقع السياحة البيئية وذات

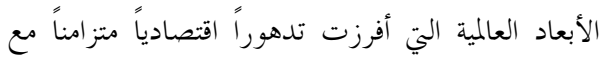

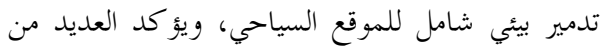

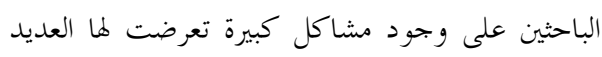
من المواقع السياحية على مدى العقود السابقة يعود إلى مدالى

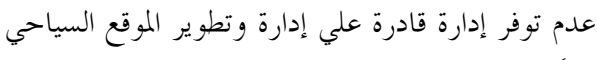

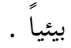
r. الضغط المتزايد على المواقع السياحية والذي أفرز استغلال متز ايد للمصادر الطبيعية وعدم التوازن الصحيح الديح مع البيئة والذي أدى إلى حدوث عدد لمديد كبير من المشاكل

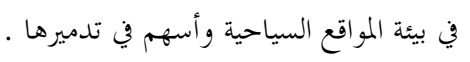

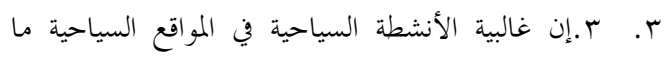

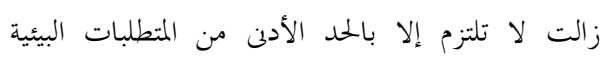

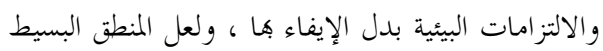

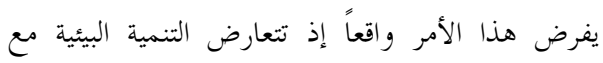




$$
\begin{aligned}
& \text { وبذلك تلبي هذه المواصفة توافق مستدام بين البيئة السياحية والمتمع }
\end{aligned}
$$

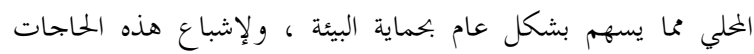

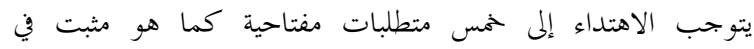

$$
\begin{aligned}
& \text { المواصفة } 14001 \text { ISO وهي : الاهني } \\
& \text { 1. السياسة البيئية }
\end{aligned}
$$

r. التخطيط : ويضم : القضايا (المحاور) البيئية ، المتطلبات

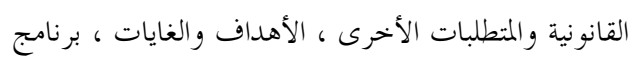

$$
\text { (برامج) الإدارة البيئية. }
$$

r. التنفيذ والتشغيل : ويضم : الهيكلية والمسؤوليات ، التئية

التدريب ، الوعي ،المهارات ، الاتصالات ، توثيق نظام

الإدارة البيئية ، مراقبة الوثائق ، المراقبة التشغيلية (العملياتية)

$$
\text { وتوقعات الطوارئ وردود الفعل. }
$$

ع. التفحص وإجراءات التصحيح : وتضم : الكشف ودرد الفيل

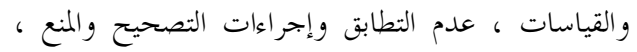

$$
\text { السجلات ، تدقيق نظام الإدارة البيئية. }
$$

$$
\text { ه. - ما ماجعة الإدارية }
$$

وكل متطلب من هذه المتطلبات نال شرحاً وافياً داخل المواصفة المارية

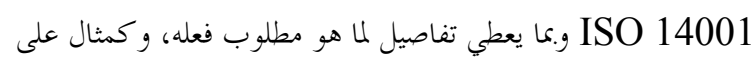
ذلك نأخذ أحد المتطلبات وهو الأهداف والغايات (Sobjectives)

.Feigenbaum 2003) (and Targets

$$
\text { إذ تشرح المواصفة } 14001 \text { ISO هذا البند بالآتي : }
$$

أ. يجب على المنشآت السياحية في المحميات الطبيعية إقامة وإدامة

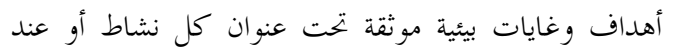

مستوى واحد أو أكثر من المستويات الإدارية في المنشأة

$$
\text { السياحية. }
$$

ب. عند إقامة ومر اجعة أهداف المنشأة السياحية يجب الأخذ بعين

الاعتبار الاطر القانونية والمتطلبات الأخرى مثل القضايا البيئية

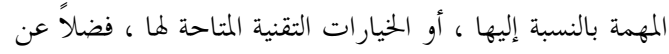

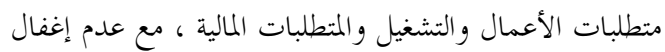

وجهات نظر الجهات ذات العلاقة (Goldsack 2008).

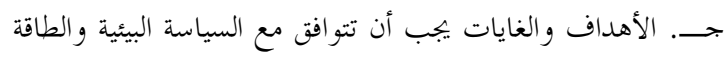

الاستيعابية في الموقع · الافل

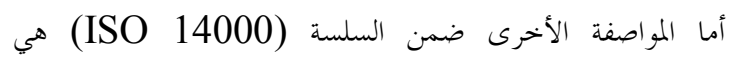

المواصفة الدليل (المواصفة الإرشادية) ISO 14004 (نظم الإدارة

البيئية ، أدلة عامة عن مبادئ نظم التقنية الساندة لإدارة البيئة)
ه. المواصفة ISO 14024 : العلامات والتصريحات البيئية -

البرامج المهنية- مبادئ توحيد إجراءات الشهادة والتطبيقات

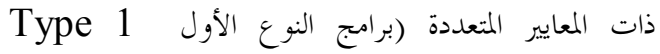

.(program

T. المواصفة ISO ISO أدوات تقيم الأداء البيئي والتدقيق

Environmental Performance البيئي

.Evaluation

V. المواصفة ISO ISO أدوات تقييم دورة الحياة البيئية

Environmental Life cycle Evaluation

(مبادئ وصيغ توجيهية).

^. المواصفة ISO IS041 : أدوات تقييم دورة الحياة البيئية :

الهدف والتعريف ، الخحال وتقييم الحزين.

9. المواصفة ISO IS042 أدوات تقييم دور الحياة البيئية :

تفسير دورة الحياة (Impact Evaluation).

• ا. المواصفة ISO أدوات تقييم دورة الحياة البيئية : 14043 :

تفسير دورة الحياة (Interpretation).

II المواصفة ISO ISO المصطلحات والتعريفات : الدليل

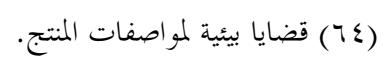

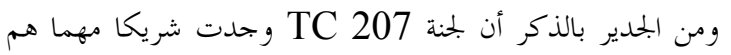
لجنة (ISO) لشؤون الدول النامية (DEVCO) هدفها المساعدة

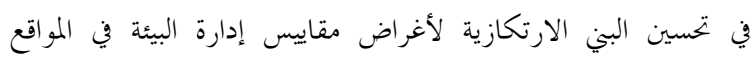

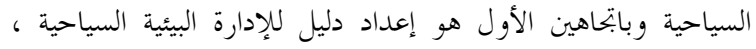

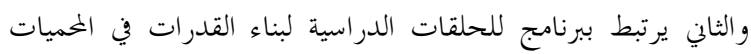

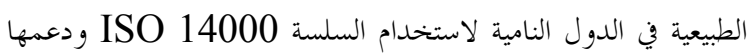

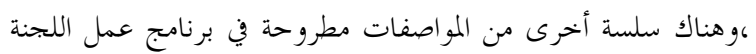
ترتبط بدليل الإدارة البيئة للمواقع السياحية صغيرة

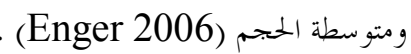
وتعد المواصفة ISO 14001 (نظم الإدارة البيئية - المواصفات

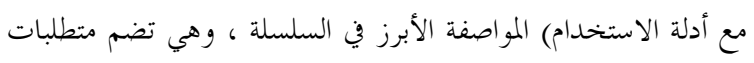

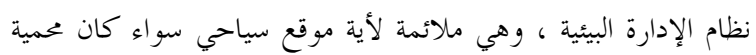
طبيعية ، فندق أم متتجع وهي قدف إلى الئه 1. أستدامة وتحسين نظام الإدارة البيئية في المواقع السياحية.

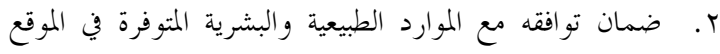
السياحي. r. التسجيل ومنح الشهادة، من خلال طرف دولي محايد. . 


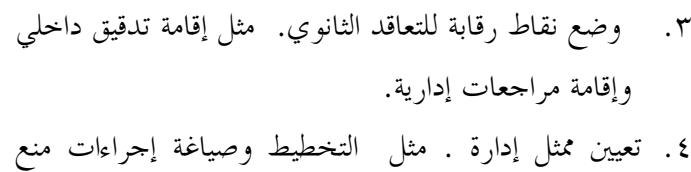

وتصحيح.

ه. فحص وتقييم دوري لنقاط المراقبة التشغيلية الأساسية.

ويشمل التطابق مع القوانين والمواصفات و المتطلبات الأخرى والمعايرة . المكل

إلا أن السلسة ISO 14000 تمتاز بالوضوح الأكبر فيما يرتبط

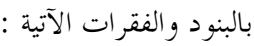
ا. ا. وضع الأهداف والغايات المتفق عليها. r. T. الاستعداد للمواقف الطارئة. r. الإعلان عن السياسة البيئية في الموقع السياحي. ع. الأخذ بعين الاعتبار وجهات نظر كل الأطراف ذات العلاقة.

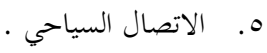

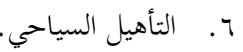

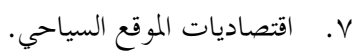

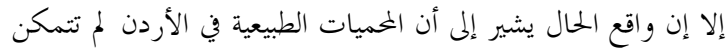

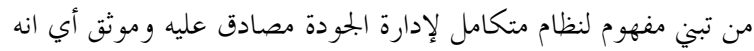

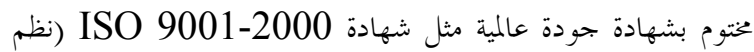
إدارة وضمان الجودة ).و بالتالي فهي لا تستطيع أن تحقق التكامل بين

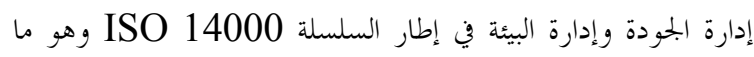

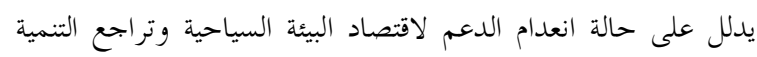
السياحية المستدامة والضغط على الموارد السياحية (1996 Sierra).

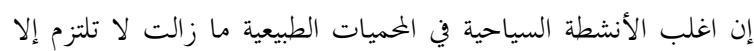

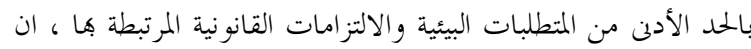
مؤشرات الواقع لا تدع للمحميات الطبيعية بدائل عدة بل بديلاً واحداً

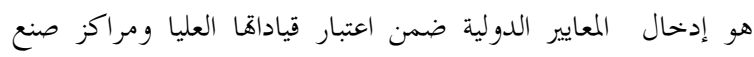

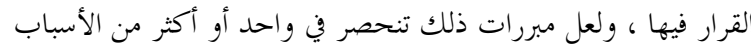

1. التحولات التي طرأت على الحركة السياحية العالمية فهو

يعتبر الموقع السياحي ذا جودة عالية عند تطابق مواصفات النوعية مع المواصفات البيئية دون إحداث أي ضرر للبيئة.

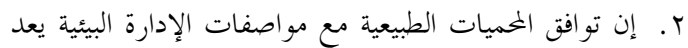

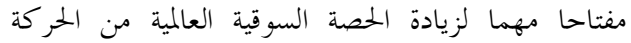

(Mike 2007) وهذه المواصفة غنية بالأفكار والمبادئ والاقتراحات والأقسام المساعدة التطبيقية ، التي تشمل نماذج عملية لما تختاجه المحميات الطبيعية عند التقدم للحصول على شهادة بإحدى مواصفات

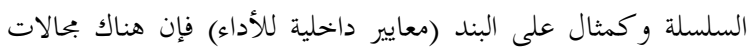
ومواقع يمكن للمحمية الطبيعية أن تقيم معايير أداء داخلية فيها مثل نظم الإدارة، مسؤولية العاملين، التعيينات، إدارة الملكية، البهزين،

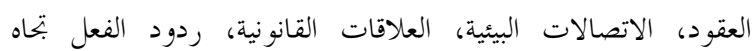

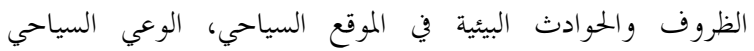

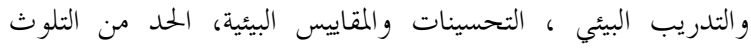

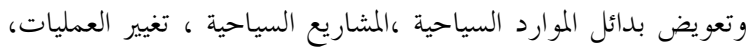

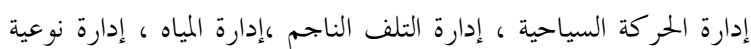

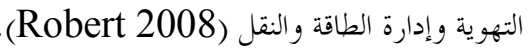

ثالثاً : الإمكانات المتاحة أمام المحميات الطبيعية في

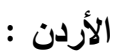
لمعالجة هذا المور نطرح التساؤلات الآتية :

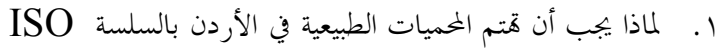
r. وما هو المطلوب عمله من الأطراف ذات العلاقة لكي تستطيع هذه الخميات الطبيعية الحصول على شهادة 14001 ISO وبالتالي تكون صديقة للبيئة؟ وقبل الإجابة عن هذه التساؤلات يثار تساؤل آخر هو : - مل تلعتمد الخميات الطبيعية في الأردن نظاماً موثقاً ومصادقاً

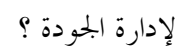

إن السؤال الأخير مقصود إذ أن وجود ذلك النظام يسهل من جهة

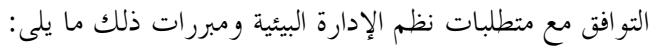

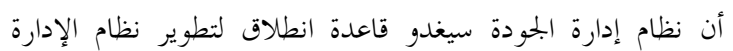

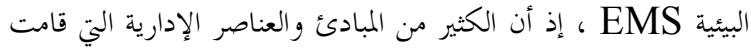

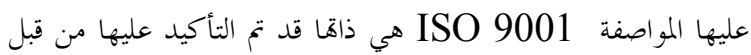
واضعي السلسلة ISO 14000 والخاصة بالبيئة ( Sacks

$$
\begin{aligned}
& \text { 2000)، ومن هذه المبادئ نذكر : والمعا }
\end{aligned}
$$

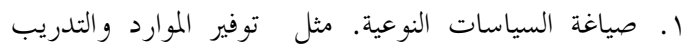

$$
\begin{aligned}
& \text { ومراقبة العمليات التشغيلية. }
\end{aligned}
$$

r. ت توثيق النظام. مثل إقامة إجراءات مراقبة الوثائق والاحتفاظ

$$
\text { بالسجلات وإدامتها. }
$$


البيئية والأداء البيئي والمعبّر عنها بعشرين فقرة في استمارة الإستبانة

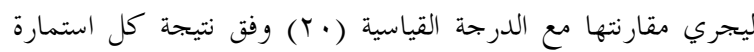
لاختبار ( T ) وطبقاً للفر ضية الصفرية:

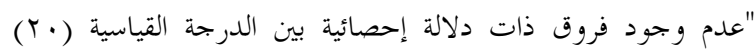

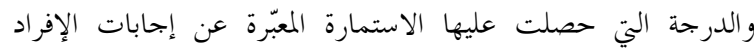
العاملين في الخمميات الطبيعية أمام المتغيرات (البنود)".

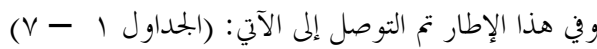

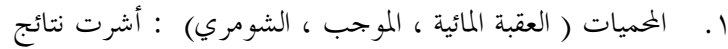

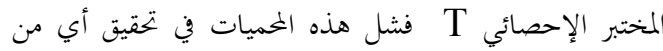

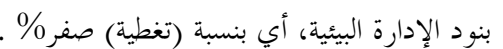

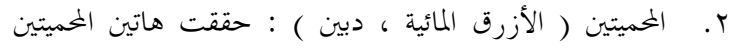

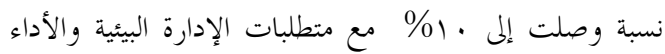

البيئي r. محمية البتراء حققت نسبة وصلت إلى 01\% \%

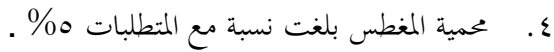

\begin{tabular}{|c|c|c|c|c|}
\hline$W_{1}$ & $\mathbb{N}$ & & S.T. & $T * *$ \\
\hline $\mathrm{VI}$ & 10 & & 1033 & 1592 \\
\hline 12 & 10 & & 2025 & 765 \\
\hline 74 & 10 & & 0943 & 436 \\
\hline VS & 10 & & 3824 & 562 \\
\hline V6 & 10 & & 4351 & 6.10 \\
\hline$n$ & 10 & & 4.185 & 393 \\
\hline V11 & 10 & & 4223 & 4.12 \\
\hline $\mathrm{V} 13$ & 10 & & 4968 & 427 \\
\hline 714 & 10 & & 5996 & 327 \\
\hline V15 & 10 & & 4.402 & 4.74 \\
\hline प17 & 10 & & S.48! & 427 \\
\hline 718 & 10 & & 4.725 & 542 \\
\hline$\nabla 3$ & 10 & & 0843 & 28.50 \\
\hline 18 & 10 & & 3213 & 482 \\
\hline 719 & 10 & & 487 & 564 \\
\hline 19 & 10 & & 2348 & 431 \\
\hline V16 & 10 & & 2908 & 5.11 \\
\hline 120 & 10 & & 2497 & 4.18 \\
\hline vio & 10 & & 391 & 421 \\
\hline $\mathrm{V} 12$ & 10 & & 1524 & 6.43 \\
\hline Cloot & Verty & Heb & Lood & Save \\
\hline
\end{tabular}

الجدول (1) نتائج التحليل الإحصائي Tلإجابات أفراد العينة في محمية دبين.

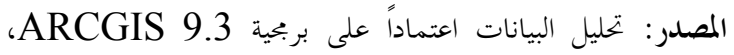
وجاء ترتيب Vi (البنود) على وفق الإدخال والمعالجة ، وكذا الحال

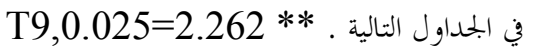

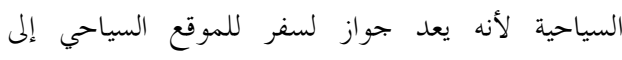
الأسو اق الدولية أولا ومؤشراً إيهابيا ثانياً.

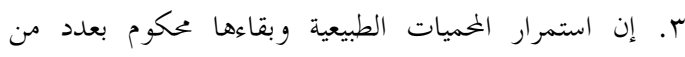
العوامل يتقدمها عامل التوازن مع البيئة السياحية ومواردها الطبيعية.

إذ لا يككن لأي موقع سياحي تنموي أن ينهض في ظل بيئة متدهورة وهو ما يعد سببا للاهتمام الواسع النطاق بالمواصفة الدولية القياسية ISO 14001 1. مواجهة الخمميات الطبيعية الأردنية ارتفاعا واضحا في كلف

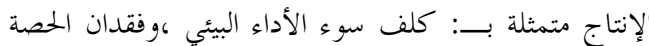
السوقية بسبب عزوف السياح عن الموقع السياحي ذا الأداء البيئي المتدهور من جهة ، وانغفاض مستوى الجودة لمنتجاته وخدماته من جهة ثانية ، فضلاً عن انعدام الصورة الذهنية

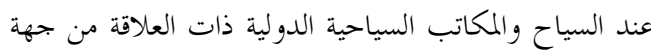
ثالثة r. الضغوطات التي تمارسها المنظمات والبحالس المهتمة بشؤون البيئة للحد من الثلوث الذي يلزم المواقع السياحية المختلفة معالجة الأضرار البيئية الحادثة. r. احتمالات المستقبل المخيفة التي تؤشر بداية نضوب الموارد الطبيعية وتدميرها في المواقع السياحية بسبب الآثار البيئية

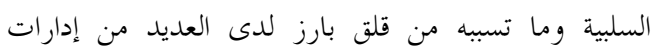
الخميات الطبيعية .

رابعاً : متطلبات تحسين الإدارة البيئية والأداء البيئي في البيدية البيات المحميات الطبيعية الطية لتغطية هذا الحور تم توزيع استمارة الاستبانة ذات العشرون سؤالاً

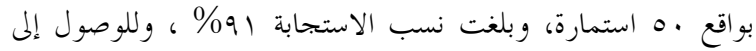
النتائج وتقديم الاستتاجات التطبيقية تم الاعتماد على التحليل الإحصائي ( T ) إذ اعتمد الباحث على معالجة البنود الرئيسة للإدارة 
Journal of Environmental Studies [JES] 2010. 4: 59-68 Article

\begin{tabular}{|c|c|c|c|}
\hline$V_{i}$ & 14 & St: & $T^{* 1}$ \\
\hline $\mathrm{VI}$ & 10 & 406 & 265 \\
\hline$V_{2}$ & 10 & 3.2 & 2.67 \\
\hline V4 & 10 & 295 & 11.14 \\
\hline V5 & 10 & 4.38 & 354 \\
\hline V6 & 10 & 509 & 3.67 \\
\hline V7 & 10 & 5.03 & 6.16 \\
\hline V11 & 10 & 4.65 & 3.13 \\
\hline$v 13$ & 10 & 392 & 3.79 \\
\hline V14 & 10 & 396 & 3.92 \\
\hline V15 & 10 & 450 & 4,5 \\
\hline $\mathrm{V} 17$ & 10 & 459 & 262 \\
\hline V18 & 10 & 4.22 & 4.27 \\
\hline V3 & 10 & 345 & 5.41 \\
\hline V8 & 10 & 282 & 4.71 \\
\hline V19 & 10 & 3.40 & 902 \\
\hline V9. & 10 & 2898 & 4.15 \\
\hline V16 & 10 & 2821 & 4.71 \\
\hline$\sqrt{20}$ & 10 & 2550 & 558 \\
\hline V10 & 10 & 474 & 4 \\
\hline$V_{12}$ & 10 & 1252 & 6.82 \\
\hline Clos & Verity & Losd. & Save. \\
\hline
\end{tabular}

الجدول(ب) نتائج التحليل الإحصائي Tلإجابات أفراد العينة في محمية البتراء.

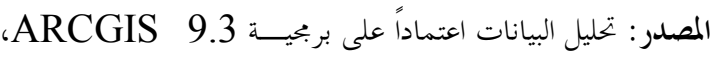
T9,0.025=2.262**

\begin{tabular}{|c|c|c|c|}
\hline$V_{i}$ & $\mathrm{~N}$ & S.T. & $T * *$ \\
\hline V1 & 10 & 2.923 & 3.14 \\
\hline V2 & 10 & 2.011 & 5.66 \\
\hline V4 & 10 & 3.307 & 7.08 \\
\hline V5 & 10 & 2.160 & 10.25 \\
\hline V6 & 10 & 3.164 & 3.7 \\
\hline V7 & 10 & 1.506 & 9.66 \\
\hline V11 & 10 & 1.703 & 5.01 \\
\hline V13 & 10 & 3.190 & 5.75 \\
\hline V14 & 10 & 2.214 & 5.29 \\
\hline V15 & 10 & 2.111 & 3.45 \\
\hline V17 & 10 & 3.795 & 5.67 \\
\hline V18 & 10 & 3.406 & 6.13 \\
\hline V3 & 10 & 2.366 & 7.48 \\
\hline V8 & 10 & 1.874 & 4.73 \\
\hline V19 & 10 & 3.342 & 4.26 \\
\hline V9 & 10 & 1.430 & 5.75 \\
\hline V16 & 10 & 2.369 & 3.34 \\
\hline $\mathrm{V} 20$ & 10 & 1430 & 5.75 \\
\hline V10 & 10 & 1.647 & 8.45 \\
\hline V12 & 10 & 0.966 & 4.58 \\
\hline Cleas & Verity & Help Lasd. & Save. \\
\hline
\end{tabular}

الجدول(ه) نتائج التحليل الإحصائي الإجابات أفراد العينة في محمية الموجب.

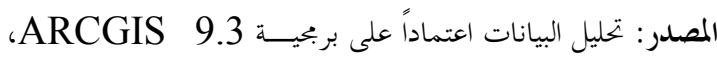
T9,0.025=2.262**

\begin{tabular}{|c|c|c|c|c|}
\hline Vi & N & S.T. & $T^{* * *}$ \\
\hline V1 & 10 & 4.01 & 4.18 \\
\hline V2 & 10 & 0.97 & 13.69 \\
\hline V4 & 10 & 0.42 & 9.09 \\
\hline V5 & 10 & 4.39 & 3.15 \\
\hline V6 & 10 & 2.67 & 3.55 \\
\hline V7 & 10 & 3.98 & 36.37 \\
\hline V11 & 10 & 0.67 & 6.89 \\
\hline V13 & 10 & 0.70 & 3.80 \\
\hline V14 & 10 & 0.48 & 3.02 \\
\hline V15 & 10 & 0.48 & 7.10 \\
\hline V17 & 10 & 0.48 & 7.02 \\
\hline V18 & 10 & 0.97 & 3.29 \\
\hline V3 & 10 & 1.932 & 3.14 \\
\hline V8 & 10 & 1.265 & 6.89 \\
\hline V19 & 10 & 2.224 & $2.43^{*}$ \\
\hline V9 & 10 & 0.422 & 2.74 \\
\hline V16 & 10 & 0.707 & 10.16 \\
\hline V20 & 10 & 0.669 & $2.54^{*}$ \\
\hline V10 & 10 & 1.135 & 3.07 \\
\hline V12 & 10 & 0.483 & 3.16 \\
\hline Clos & Venify & Help & Losd & Save \\
\hline
\end{tabular}

الجدول(؟) نتائج التحليل الإحصائي T لإجابات أفراد العينة في محميسة الأزرق المائية

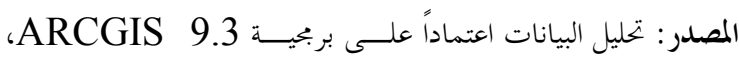

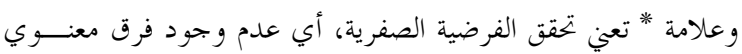
T9,0.025=2.262**

\begin{tabular}{|c|c|c|c|c|}
\hline$W_{i}$ & 11 & \multicolumn{2}{|c|}{ ST } & $I=1$ \\
\hline YI & 8 & \multicolumn{2}{|c|}{0535} & 265 \\
\hline$y z$ & y & \multicolumn{2}{|c|}{150} & $2: 8$ \\
\hline V4 & a & \multicolumn{2}{|c|}{1168} & 863 \\
\hline Ws & 8 & \multicolumn{2}{|c|}{1723} & 38 \\
\hline$W$ & 8 & \multicolumn{2}{|c|}{1600} & 251 \\
\hline y. & y & \multicolumn{2}{|c|}{1949} & 302 \\
\hline W1 & 8 & \multicolumn{2}{|c|}{0726} & 3,5 \\
\hline vI3 & 8 & \multicolumn{2}{|c|}{0745} & 4.81 \\
\hline Vit & 7 & \multicolumn{2}{|c|}{0535} & 265 \\
\hline Vis & g & \multicolumn{2}{|c|}{0707} & 865 \\
\hline Wh & 8 & \multicolumn{2}{|c|}{0926.} & $30 \mathrm{~b}$ \\
\hline vis & 8 & \multicolumn{2}{|c|}{1581} & 313 \\
\hline 13 & 8 & \multicolumn{2}{|c|}{074} & 487 \\
\hline w & f & \multicolumn{2}{|c|}{1300} & 324 \\
\hline Wa & 8 & \multicolumn{2}{|c|}{070} & $1^{2}$ \\
\hline w & $\theta$ & \multicolumn{2}{|c|}{0756} & 387 \\
\hline Vis & 8 & \multicolumn{2}{|c|}{045} & 3.6 \\
\hline 70 & 8 & \multicolumn{2}{|c|}{0535} & 1.65 \\
\hline Yo & 8 & \multicolumn{2}{|c|}{0786} & $187 *$ \\
\hline Cleat & Verity & Heb & Losd. & Save. \\
\hline
\end{tabular}

الجدول(\&) نتائج التحليل الإحصائي T لإجابات أفراد العينة في محمية العقبة المائية.

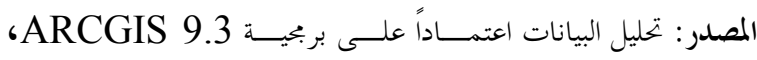
$\mathrm{T} 7,0.025=2.365 * *$ 
Journal of Environmental Studies [JES] 2010. 4: 59-68 Article

\begin{tabular}{|c|c|c|c|c|}
\hline$V_{i}$ & $\mathrm{~N}$ & & T. & $T * *$ \\
\hline V1 & 8 & & 66 & 3.69 \\
\hline V2 & 8 & & 38 & 3.61 \\
\hline V4 & 8 & & 64 & 27.34 \\
\hline V5 & 8 & & 2 & 6.36 \\
\hline V6 & 8 & & 74 & 4.05 \\
\hline V7 & 8 & & 21 & 7.94 \\
\hline V11 & 8 & & 96 & 4.83 \\
\hline V13 & 8 & & 82 & 3.33 \\
\hline V14 & 8 & & 42 & 4.55 \\
\hline V15 & 8 & & 72 & 27.02 \\
\hline V17 & 8 & & 37 & 4.37 \\
\hline V18 & 8 & & 65 & 79.4 \\
\hline V3 & 8 & & 55 & 4.83 \\
\hline V8 & 8 & & 88 & 3.68 \\
\hline V19 & 8 & & 7 & $1.53^{*}$ \\
\hline V9 & 8 & & 63 & 4.14 \\
\hline V16 & 8 & & 76 & 4.35 \\
\hline V 20 & 8 & & 65 & 4.45 \\
\hline V10 & 8 & & 17 & 3.21 \\
\hline V12 & 8 & & 89 & 4.58 \\
\hline Clear & Verity & Hetp & Losd. & Save. \\
\hline
\end{tabular}

الجدول(V) نتائج التحليل الإحصائي الإجابات أفراد العينة في محمية المغطس.

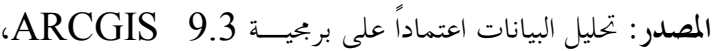

$\mathrm{T} 7,0.025=2.365^{* *}$

يملكها الكادر الوظيفي إلا إن التقارب متحقق ميدانياً من خحلال النظم الإدارية والمالية المعتمدة.

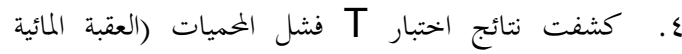
والموجب والشومري )، من تحقيق أي توافق مع البنود العشرين للإدارة البيئية.

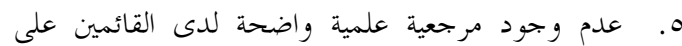
إدارة المحميات الطبيعية لأسس الإدارة البيئية السليمة وحتهية الواجب إتباعها في إدارة وتطوير المحميات الطبيعية

ثانياً : التوصيات. بغية استكمال ضرورات الدراسة الميدانية، يتناول هذا المحور عدد من التوصيات والمقترحات اعتماداً على نتائج التحليلات الإحصائية وباتحاهات متعددة منها ما يساعد إدارات الخميات الطبيعية المبحوثة في التوافق مع مستوى أداء بيئي سياحي مناسب ومنها ما يرتبط

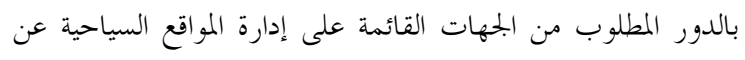

\begin{tabular}{|c|c|c|c|}
\hline$W_{1}$ & 4 & $5 \pi$ & $T *$ \\
\hline$\forall$ & 6 & 1.36 & 4.16 \\
\hline$V_{2}$ & 6 & 0.894 & 11169 \\
\hline$V_{4}$ & 6 & 200 & 909 \\
\hline W & 6 & 4412 & 315 \\
\hline VE & 6 & 5750 & 355 \\
\hline 77 & 6 & 0.915 & 3637 \\
\hline VII & 6 & 2251 & 689 \\
\hline V13 & 5 & 3225 & 3.80 \\
\hline V14 & b. & 4360 & 302 \\
\hline vis & 5 & 2875 & 710 \\
\hline Vi7 & 6 & 1862 & 702 \\
\hline VIs & 5 & 4.472 & 329 \\
\hline 73 & 6 & 34 & 314 \\
\hline ye. & b & 24 & 639 \\
\hline tis & 6 & 900 & $243^{4}$ \\
\hline W & 6 & 789 & 274 \\
\hline V/ 6 & 5 & 1765 & 1016 \\
\hline vo & 6 & 2251 & 254 \\
\hline VIo & 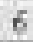 & 322 & 307 \\
\hline V12 & 5 & 1.49 & 316 \\
\hline Cless & & Heb Las & Save. \\
\hline
\end{tabular}

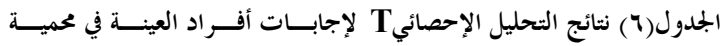

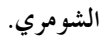
المصدر : تحليل البيانات اعتماداً على بربجيــة ARCGIS 9.3 9.3 $\mathrm{T} 5,0.025=2.571 * *$

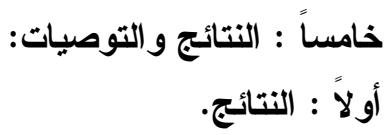

1. أكد البحث على إمكانية استثمار وتطبيق البنود العشرين

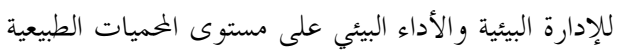

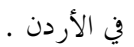

r. كشفت نتائج اختبار T أن محمية الأزرق المائية حققت

نسبة توافق مع بنود الإدارة البيئية متساوية مع محمية ديين

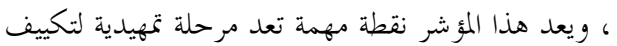

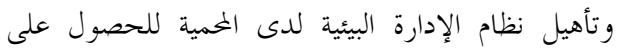

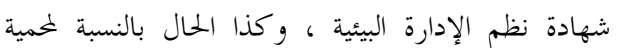

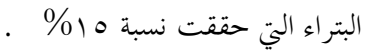
r. وعلى الرغم من التوصل إلى بعض الاختلافات بين تلك

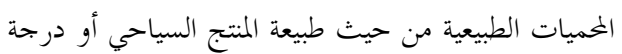
مرونة الطلب أو طبيعة المؤهلات والاختصاصات التي ميني مئي 
ب. إصدار المواصفات والمقاييس الحناصة بإدارة وتطوير

مواقع السياحة البيئية.

ه. بالنسبة إلى الخميات الطبيعية :

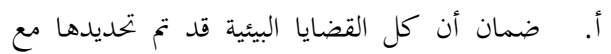

صياغة واضحة لمواصفات رقابة فاعلة لمنع وتقليل

التأثيرات البيئية السلبية الختملة.

ب. تعريف العاملين في المحميات الطبيعية وقبل ذلك

شحذ همم ودعم الإدارات العليا فيها نحو طرائق

أداء الأنشطة المؤثرة في البيئة مع جعل هذه إلمسالة

(المسالة الأولى) في تفكير هؤلاء.

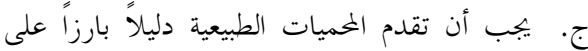

التحسينات البيئية التي ساهمت فيها بشكل مباشر

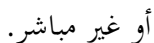

7. . بالنسبة إلى الجهات الأخرى:

أ. تصميم وتنفيذ حملة إعلامية شاملة ومستمرة تعتمد المد

كل وسائل الاتصال (انترنت، ملصقات، راديو، ولثيل

تلفزيون، ندوات، مقالات ) ، من أجل حشد أرس

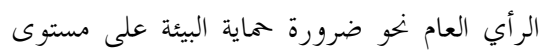

الفرد والجماعة .

ب. الحيلولة دون تشتت المسؤولية القضائية على قضايا

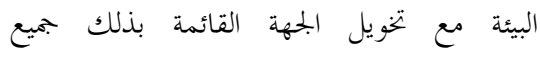

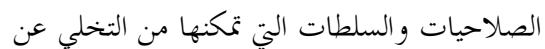

الإجراءات العلاجية بابتاه إجراءات وقائية فاعلة.

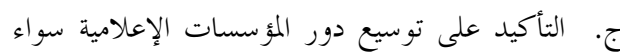

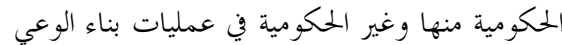

البيئي مؤسسياً وجماهيرا وعلى الأصعدة والمحالات

كافة. - مافي

د. التعاون والتنسيق بين الوزارات و الدوائر ذات

الاختصاص في التعرف على المخاطر البيئية التي تواجه

المواقع السياحية ومصادرها من اجل السيطرة عليها .

ومن الجحدير بالذكر أن بحاح الإجراءات سابقة الذكر لا يتم إلا في

: إطار

1. التنسيق المباشر بين مختلف أطراف صناعة السياحة في

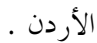

إقرار خطط التنمية السياحية، وثالثة ترتبط بجهات ذات علاقة بالإدارة البيئية والجهات المساندة وعلى وفق الآتي :

1. يوصي الباحثان إدارة المحميات الطبيعية موضوع الدراسة

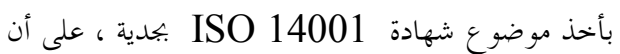
لا تكون الجهود والإجراءات بحرد رد فعل لتوجيهات عليا من قبل الوزارة أو الهيئات ذات العلاقة، فالاقتناع الكامل

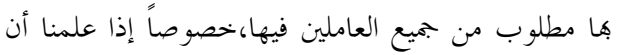
من بين مؤشرات تقويم الأداء السياحي المحافظة على البيئة وتحسين استدامتها .

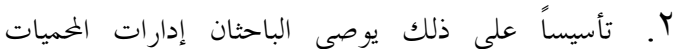
الطبيعية المبحوثة جميعاً بضرورة السعي نحو الإسراع في إقامة المتطلبات و البنود الخاصة بشهادة 14001 ISO

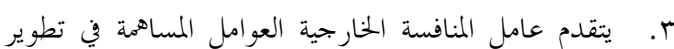
نظم العمل في المحمية الطبيعية، والتي سيتحقق عندها الأثر التتابعي لشهادة ISO، حيث يحفز الدخول إلى الأسواق

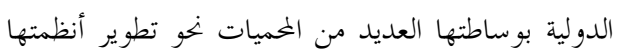

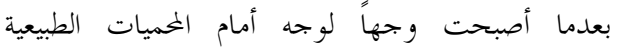

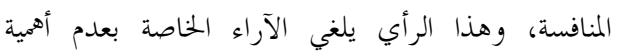

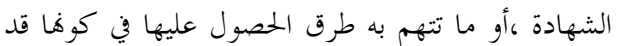
تخلو من الحياد والأمانة، لأن المحمية الطبيعية تكون أمام

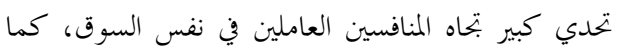
يتحقق الأثر التتابعي التطويري للشهادة من خلاهل تشديد

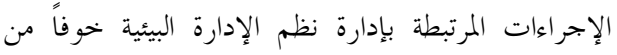

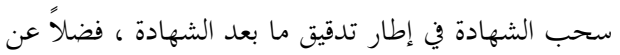

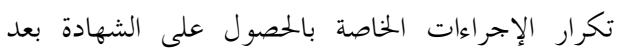
مرور r سنوات. ع. التوصيات الخاصة بالجهات ذات العلاقة بالإدارة البيئية

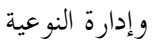
أ. تأهيل جهة خاصة في الأردن للإشراف على تفويض بمنح الشهادة بإحدى مواصفات السلسلة ISO وقبلها السلسلة ISO 14000 ، ليس فقط في المحميات الطبيعية وإنما يشمل هذا مختلف المواقع والمنشآت السياحية والفندقية في

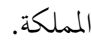


Enger F.H. 2006. "ISO 9000 \& ISO 14000, Compatibility Challenges \& Hurdles" ISO Bulletin, August.

Feigenbaum A.V. 2003. Quality \& Business Growth Today, Quality Progress, Nov.

Goldsack B. 2008. "Going global with ISO 14001", Review, December.

Mike F. 2007. "Quality Assurance" Information Mgmt. Jour., Vol.6, Iss. 2, Winter.

Robert W. 2008. Integrating ISO 14000 \& ISO 9000 into one system, Pollution Engineering, June.

Sacks I. 2000." The Right To Sustainable Development "Label, France, April.

Sierra. E. 1996. The new ISO 14000 Series: What Exporters Should Know, International Trade Forum, 3 .

$$
\begin{aligned}
& \text { r. التنسيق بين الإدارة البيئية وإدارة النوعية على مستوى } \\
& \text { الخمية الطبيعية والأجهزة ذات الاهتمام المشترك. } \\
& \text { وبذلك تتوفر فرصة كبيرة للمحميات الطبيعية في الأردن لمواكبة }
\end{aligned}
$$

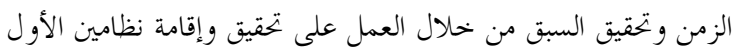

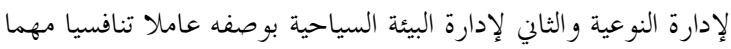

$$
\begin{aligned}
& \text { وحيويا في ترويج البرامج السياحية في الأسواق الدولية. } \\
& \text { المر اجع }
\end{aligned}
$$

Carman J.M. 2007. Continuous Quality Improvement As A Survival Strategy: The Southern Pacific Experience" California Mgmt. Review, Vol. XXVII,No.12,

Dixon J. 2005. Environment: ISO / TC 207 Spreads the News, ISO Bulletin, Oct. 2005. Elmuti D, Kathawala Y. 2008. An Investigation Into The Effects Of ISO9000 On Participant's Attitudes \& Job Performance" Production \& Inventory Mgmt. Jour., Second Quarter.

\title{
Environmental Performance Auditing (ISO 14001) In Natural Resave in Jordan and It's Impact On Minimization of Environmental Risks (case Study)
}

\section{Ibrahim K. bazazo}

Associate Professor, Head of tourism and marketing dept. Faculty of Business Middle East University Mob- +962-7-77224468 fax: +962-6-4129613

E-mail: Ibazazo@meu.edu.jo

\begin{abstract}
This study seeks to explore the preparations for ISO 14001- in industrial tourism as case study of natural resave in Jordan. The level of fitness with the requirements of these specification and measure the effect of that level to minimize the environmental risks was analyzed. Questionnaire model was used to test the hypotheses. The study resulted in a number of valuable results, and introduced some conclusions, which became a base to help the managers of natural resave to overlap the environmental risks. The Study covered five sections starting with identify the ISO 14001 and the concepts of it's exists. Also, the opportunities of natural resave for improving its environmental performance were determined.
\end{abstract}

Key words: ISO 14001. Ecotourism. Industry Tourism and Environmental Performance 